\title{
AN EQUICONTINUITY CONDITION FOR TRANSFORMATION GROUPS
}

\author{
JOHN D. BAUM
}

The purpose of this paper is to extend an unpublished theorem of Kakutani, which gives a necessary and sufficient condition for equicontinuity in dynamical systems. We shall state and sketch the proof of Kakutani's theorem and then state and prove the generalization thereof.

1. Definitions. These definitions are essentially those given by Gottschalk and Hedlund (cf. [3]). ${ }^{1}$ Let $X$ be a topological space, $T$ a topological group with identity $e$, and $\pi$ a mapping of $X \times T$ into $X$ with the properties: (1) $\pi(x, e)=x$, (2) $\pi\left(\pi\left(x, t_{1}\right), t_{2}\right)=\pi\left(x, t_{1} t_{2}\right)$, (3) $\pi$ is continuous. The triple $(X, T, \pi)$ is called a transformation group (or dynamical system). Henceforth we shall write $\pi(x, t)$ simply as $x t$; and if $A \subset T$ then $x A=\{x t \mid t \in A\}$. The orbit of $x$ is the set $x T$; the orbit closure of $x$, the set $\mathrm{Cl}(x T)$. The set $A$ is said to be minimal under $T$ or simply minimal, provided $A$ is an orbit closure and $A$ does not properly contain an orbit closure.

In what follows we shall be dealing with uniform spaces; for the properties of such spaces we refer to [4]. We alter the notation in writing $x \alpha$ instead of $V_{\alpha}(x)$ for "the neighborhood of $x$ of index $\alpha$." The group $T$ is called equicontinuous at $x \in X$, provided the collection of mappings $\left\{\pi^{t} \mid t \in T\right.$, where $\left.\pi^{t}(x)=x t\right\}$ is equicontinuous at $x$, i.e. for each index $\alpha$ of $X$ there exists an index $\beta$ of $X$ such that $x \beta t \subset x t \alpha$ for all $t \in T$. The group $T$ is called equicontinuous provided it is equicontinuous at each point of $X$. The group $T$ is called uniformly equicontinuous provided the collection of mappings $\left\{\pi^{t} \mid t \in T\right\}$ is uniformly equicontinuous, i.e. for each index $\alpha$ of $X$ there exists an index $\beta$ of $X$ such that $x \beta t \subset x t \alpha$ for all $t \in T$ and all $x \in X$.

Let $T$ be a topological group and let $A \subset T$, then $A$ is said to be left (right) syndetic in $T$ provided that $T=A K(T=K A)$ for some compact subset $K$ of $T$. If $T$ is abelian these two notions coincide, and we simply say that $A$ is syndetic. The point $x \in X$ is said to be almost periodic under $T$ provided that for each index $\alpha$ of $X$, there exists a left syndetic subset $A$ of $T$ such that $x A \subset x \alpha$. A point $x \in X$ is said to be discretely almost periodic under $T$ provided that for each index $\alpha$ 1952.

Presented to the Society, April 25, 1953; received by the editors December 20 ,

1 Numbers in brackets refer to the references cited at the end of the paper. 
of $X$ there exists a set $A$ in $T$ and a finite set $F$ in $T$ such that $T=A F$ and $x A \subset x \alpha$ (i.e. $x$ is almost periodic relative to the discrete topology in $T$ ). The group $T$ is said to be almost periodic provided that for each index $\alpha$ of $X$, there exists a left syndetic subset $A$ of $T$ such that $x A$ $\subset x \alpha$ for all $x \in X$. The group $T$ is said to be discretely almost periodic provided that for each index $\alpha$ of $X$, there exists a set $A$ in $T$ and a finite set $F$ in $T$ such that $T=A F$ and $x A \subset x \alpha$, for all $x \in X$ (i.e. $T$ is almost periodic relative to its discrete topology).

Let $Y$ be a topological space, $X$ a uniform space, and let $\Phi$ be a class of mappings of $Y$ into $X$. Let $\alpha$ be an index of $X$, define $\alpha^{*}$ $=\{(\phi, \psi) \mid(\phi(y), \psi(y)) \in \alpha$ for all $y \in Y\}$, and let $U$ be the uniformity of $X$; then $\left\{\alpha^{*} \mid \alpha \in \mathcal{U}\right\}$ is a uniformity base and is said to generate the space index uniformity of $\Phi$. Let $T$ be a topological group, $X$ a uniform space, and $\Phi$ the class of all the right uniformly continuous functions on $T$ to $X$; and let $\Phi$ be provided with its space index uniformity. Let $\nu: \Phi \times T \rightarrow \Phi$ be defined by $\nu(\phi, t)=\psi$, where $\psi(\tau)=\phi(t \tau)$ for all $\tau \in T$. The uniformly equicontinuous transformation group $(\Phi, T, \nu)$ is called the left uniform functional transformation group over $T$ to $X$.

\section{Kakutani's theorem.}

TheOREM (KakUtani). Let $X$ be a compact metric space, let $R$ be the real numbers, considered as a topological group under addition, with the usual topology, let $(X, R, \pi)$ be a transformation group such that $X$ is minimal, and let $f$ be a continuous mapping of $X$ into $R$. For $x^{\prime} \in X$, let $f_{x^{\prime}}$ be the function from $R$ to $R$ defined by $f_{x^{\prime}}(t)=f\left(x^{\prime} t\right)$ for all $t \in R$. Then $R$ is equicontinuous if and only if there exists a point $x^{\prime} \in X$ such that for every continuous $f: X \rightarrow R, f_{x^{\prime}}$ is a Bohr almost periodic function [1].

We sketch the proof of the sufficiency of the condition. Let $x^{\prime}$ be a point with the required property; it will be sufficient to show that $R$ is uniformly equicontinuous on the orbit of $x^{\prime}$, a set dense in $X$. Let $\epsilon>0$. The Stone-Weierstrass theorem enables us to find a set $\left\{f_{i}(x), g_{i}(x)\right\}$ $i=1,2, \cdots, N\}$ of continuous functions on $X$ to $R$ such that

$$
\left|\rho(x, y)-\sum_{i=1}^{N} f_{i}(x) g_{i}(y)\right|<\frac{\epsilon}{4} \quad \text { for all } x, y \in X,
$$

where $\rho$ is the metric in $X$. By the hypotheses of the theorem the functions $f_{i}\left(x^{\prime} t\right), g_{i}\left(x^{\prime} t\right)$ will be uniformly bounded in absolute value (by $M>0$ ) and will be Bohr almost periodic functions. Let $\epsilon^{\prime}=\epsilon / 8 M N$. Then the set of common translation numbers for these functions is 
relatively dense in $R$, i.e. there exists a number $k\left(\epsilon^{\prime}\right)>0$ such that for any real number $t$, there exists $s, 0 \leqq s \leqq k\left(\epsilon^{\prime}\right)$, such that

$$
\begin{aligned}
& \left|f_{i}\left(x^{\prime}(u+t)\right)-f_{i}\left(x^{\prime}(u+s)\right)\right|<\epsilon^{\prime}, \\
& \left|g_{i}\left(x^{\prime}(u+t)\right)-g_{i}\left(x^{\prime}(u+s)\right)\right|<\epsilon^{\prime}
\end{aligned}
$$

for all $u \in R$. Let $\delta>0$ such that $\rho(x, y)<\delta$ implies $\rho(x s, y s)<\epsilon / 4$ for all $s$ with $0 \leqq s \leqq k\left(\epsilon^{\prime}\right)$. Then if $\rho\left(x^{\prime} t_{1}, x^{\prime} t_{2}\right)<\delta$ we have

$$
\begin{aligned}
& \rho\left(x^{\prime}\left(t_{1}+t\right), x^{\prime}\left(t_{2}+t\right)\right) \\
& \leqq \\
& \left.\quad+\mid \sum_{i=1}^{N} f_{i}\left(t_{1}+t\right), x^{\prime}\left(t_{2}+t\right)\right)-\sum_{i=1}^{N} f_{i}\left(x^{\prime}\left(t_{1}+t\right)\right) g_{i}\left(x^{\prime}\left(t_{2}+t\right)\right) \mid \\
& \quad-\sum_{i=1}^{N} f_{i}\left(x^{\prime}\left(t_{1}+s\right)\right) g_{i}\left(x^{\prime}\left(t_{2}+s\right)\right) \mid \\
& \quad+\left|\sum_{i=1}^{N} f_{i}\left(x^{\prime}\left(t_{1}+s\right)\right) g_{i}\left(x^{\prime}\left(t_{2}+s\right)\right)-\rho\left(x^{\prime}\left(t_{1}+s\right), x^{\prime}\left(t_{2}+s\right)\right)\right| \\
& \quad+\rho\left(x^{\prime}\left(t_{1}+s\right), x^{\prime}\left(t_{2}+s\right)\right)<\epsilon, \quad \text { for all } t \in R .
\end{aligned}
$$

3. Generalized theorem. We now generalize Kakutani's theorem, but before we state the generalization we shall require one further definition. Let $(X, T, \pi)$ be a transformation group, let $Y$ be a uniform space, and let $f$ be a mapping $X$ into $Y$. Define $f_{x}(t)=f(x t)$ for all $t \in T$. It is clear that $f_{x}$ maps $T$ into $Y$.

3.1 PRINCIPAL THEOREM. Let $(X, T, \pi)$ be a transformation group, let $X$ be a compact $T_{2}$-space which is minimal under $T$ and let $T$ be abelian. Then $T$ is equicontinuous if and only if there exists a point $x_{0} \in X$ such that for every continuous mapping $f$ of $X$ into the real numbers, $R$, the function $f_{x_{0}}(t)$ is almost periodic in the left uniform functional transformation group over $T$ to $R$.

We must first show that $f_{x}$ is a point of $\Phi$, the class of all right uniformly continuous mappings of $T$ into $R$. We require the following lemma.

3.2 LemMA. Let $(X, T, \pi)$ be a transformation group, let $X$ be compact and let $\alpha$ be an index of $X$. Then there exists $V$, a neighborhood of $e$ in $T$, such that $x V \subset x \alpha$ for all $x \in X$.

We omit the proof since it is quite straightforward. We now show that $f_{x} \in \Phi$. In fact we prove a somewhat more general theorem. 
3.3 TheOREM. Let $(X, T, \pi)$ be a transformation group, let $X$ be compact, let $Y$ be a uniform space, and let $f: X \rightarrow Y$ be continuous. Then $f_{x}$ is a right uniformly continuous mapping of $T$ into $Y$.

Proof. Since $X$ is compact, $f$ is uniformly continuous on $X$ to $Y$. Let $\boldsymbol{\gamma}$ be an index of $Y$, and let $\delta$ be an index of $X$ such that $(x, y) \in \delta$ implies $(f(x), f(y)) \in \gamma$. By 3.2 we can find a neighborhood $V$ of $e$ in $T$ such that $v \in V$ implies $(x, x v) \in \delta$ for all $x \in X$. Thus $(f(x), f(x v))$ $\in \gamma$ for all $x \in X$ and all $v \in V$. Let $t \in T$ and let $x t=y$; then for $v \in V$, $(f(y), f(y v)) \in \gamma$ or $(f(x t), f(x t v)) \in \gamma$, or $\left(f_{x}(t), f_{x}(t v)\right) \in \gamma$. Thus for any $t \in T$ and any $s \in t V$ we have $\left(f_{x}(t), f_{x}(s)\right) \in \gamma$. This completes the proof.

We are now in a position to prove one half (the necessity) of the principal theorem. In fact, we can prove a bit more.

3.4 Theorem. Let $(X, T, \pi)$ be a transformation group, let $X$ be compact, let $Y$ be a uniform space, let $f$ be a continuous mapping of $X$ into $Y$, let $T$ be equicontinuous and abelian, and let $x \in X$. Then $f_{x}$ is an almost periodic point of $(\Phi, T, \nu)$, the left uniform functional transformation group over $T$ to $Y$.

Proof. Since $X$ is compact $T$ is uniformly equicontinuous. Gottschalk has shown [2] that this implies that $T$ is discretely almost periodic. Let $x \in X$ be fixed; by $3.3, f_{x} \in \Phi$. Since $X$ is compact, $f$ is uniformly continuous on $X$ to $Y$. Let $\Lambda$ be an index of $\Phi$, then there exists $\gamma$, an index of $Y$, such that $\gamma^{*} \subset \Lambda$; and then there exists $\delta$, an index of $X$, such that $f(x t \delta) \subset f(x t) \gamma$ for all $t \in T$. Since $T$ is almost periodic, there exists $A$, a left syndetic subset of $T$, such that for all $y \in X, y A \subset y \delta$, in particular then $x t A \subset x t \delta$ for all $t \in T$. Thus $f_{x}(t A)$ $=f(x t A) \subset f(x t \delta) \subset f(x t) \gamma=f_{x}(t) \gamma$ for all $t \in T$, whence $f_{x} A \subset f_{x} \gamma^{*} \subset f_{x} \Lambda$. This completes the proof.

The principal difficulty in the proof of our generalization of Kakutani's theorem is that since we no longer have a metric in $X$, we are no longer able to use the Stone-Weierstrass theorem to approximate it. We use the following lemma to overcome this difficulty.

3.5 Leмma. Let $(X, T, \pi)$ be a transformation group, let $X$ be compact and minimal under $T$, and let $T$ be abelian. Let $f$ be a continuous mapping of $X$ into $Y, a$ uniform space, and suppose there exists $x_{0} \in X$ such that $f_{x_{0}}$ is almost periodic in the left uniform functional transformation group over $T$ to $Y,(\Phi, T, \nu)$. Then $f_{z}$ is almost periodic for each $x \in X$, and in fact for each index $\Lambda$ of $\Phi$ there exists a syndetic subset $A$ of $T$ such that $f_{x} A \subset f_{x} \Lambda$ for all $x \in X$.

Proof. Let $\Lambda$ be an index of $\Phi$; then there exists an index $\alpha$ of $Y$ such that $\alpha^{*} \subset \Lambda$. Let $\beta$ be a symmetric index of $Y$ such that $\beta^{3} \subset \alpha$. 
Since $f_{x_{0}}$ is almost periodic, there exists a syndetic subset $A$ of $T$ such that $f_{x_{0}}(A t) \subset f_{x_{0}}(t) \beta$ for all $t \in T$, or $f\left(x_{0} A t\right) \subset f\left(x_{0} t\right) \beta$. Then for all $t_{0} \in T, f\left(x_{0} A t_{0} t\right) \subset f\left(x_{0} t_{0} t\right) \beta$, and since $T$ is abelian, we have $f\left(x_{0} t_{0} A t\right)$ $C f\left(x_{0} t_{0} t\right) \beta$ for all $t_{0}$ and $t \in T$.

Since $X$ is compact, $f$ is uniformly continuous; thus there exists an index $\gamma$ of $X$ such that $f(x \gamma) \subset f(x) \beta$ for all $x \in X$. Let $a \in A$ and $t \in T$ be fixed. Since $\pi^{t}$ and $\pi^{a t}$ are uniformly continuous, $X$ being compact, we can select a symmetric index $\delta$ of $X$ so that $x \delta t \subset x t \gamma$ and $x \delta a t \subset x a t \gamma$ for all $x \in X$. Since $X$ is minimal there exists $t_{1} \in T$ such that $x_{0} t_{1} \in x \delta$, whence $x_{0} t_{1} t \in x \delta t \subset x t \gamma$. Thus

$$
f\left(x_{0} t_{1} t\right) \in f(x t \gamma) \subset f(x t) \beta .
$$

From the first part of the proof we have

$$
f\left(x_{0} t_{1} a t\right) \in f\left(x_{0} t_{1} t\right) \beta .
$$

Now $x_{0} t_{1} \in x \delta$, and since $\delta$ is symmetric, $x \in x_{0} t_{1} \delta$; therefore $x a t$ $\in x_{0} t_{1} \delta a t \subset x_{0} t_{1} a t \gamma$, whence

$$
f(x a t) \in f\left(x_{0} t_{1} a t \gamma\right) \subset f\left(x_{0} t_{1} a t\right) \beta .
$$

From (1), (2), and (3) we have $f(x a t) \in f(x t) \beta^{3} \subset f(x t) \alpha$, and since $a$ and $t$ were arbitrary, $f(x A t) \subset f(x t) \alpha$ for all $t \in T$. Thus $f_{x} A \subset f_{x} \alpha^{*}$ $\subset f_{x} \Lambda$. This completes the proof.

We require a further lemma.

3.6 LeмMA. Let $(X, T, \pi)$ be a transformation group, let $X$ be compact, and let $T$ be abelian. Let $f$ and $g$ be continuous mappings of $X$ into $R$, the reals. Let $x \in X$ be fixed, and let $f_{x}$ and $g_{x}$ be almost periodic in $(\Phi, T, \nu)$, the left uniform functional transformation group over $T$ to $R$. Then for each $\epsilon>0$, there exists $E \subset T$ and a finite set $H C T$ such that $E H=T$ and such that $b \in E$ implies $|f(x b t)-f(x t)|<\epsilon$ and $\mid g(x b t)$ $-g(x t) \mid<\epsilon$ for all $t \in T$.

Proof. We prove that $f_{x}$ is discretely almost period. Let $\Lambda$ be an index of $\Phi$; then there exists $\alpha$, an index of $R$, such that $\alpha^{*} \subset \Lambda$. Let $\beta$ be a symmetric index of $R$ such that $\beta^{2} C \alpha$. Since $X$ is compact, $f: X \rightarrow R$ is uniformly continuous. Let $\gamma$ be an index of $X$ such that $f(x \gamma) \subset f(x) \beta$ for all $x \in X$. By Lemma 3.2 there exists $V$, a neighborhood of $e$ in $T$, such that $x V \subset x \gamma$ for all $x \in X$. Since $f_{x}$ is almost periodic, there exists $A \subset T$ and $K$, compact, in $T$ with $A K=T$, such that $f_{x} A \subset f_{x} \beta^{*}$ or $f(x a t) \subset f(x t) \beta$ for all $a \in A$ and all $t \in T$. Now $K$ is compact and $K \subset \bigcup_{k \in K} k V$; therefore there exists a finite set $\left\{k_{i}\right\}_{i=1}^{n}=K^{\prime}$ such that $K \subset K^{\prime} V$. Let $A^{\prime}=A V$; then $A^{\prime} K^{\prime}=A V K^{\prime}=A K=T$, and $A^{\prime}$ is discretely syndetic in $T$. Let $a^{\prime} \in A^{\prime}, t \in T$, and $a^{\prime}=a v$ where 
$a \in A$ and $v \in V$; then $f\left(x a^{\prime} t\right)=f(x a v t) \in f(x v t) \beta$. Also $x v t=x t v \in x t V$ $\subset x t \gamma$ whence $f(x v t) \in f(x t) \beta$; therefore $f\left(x a^{\prime} t\right) \in f(x t) \beta^{2} \subset f(x t) \alpha$ for all $a^{\prime} \in A^{\prime}$ and all $t \in T$, or $f_{x} A^{\prime} \subset f_{x} \alpha^{*} \subset f_{x} \Lambda$. This completes the proof that $f_{x}$ is discretely almost periodic. Similarly $g_{x}$ is discretely almost periodic.

Define $A(\epsilon, f)=\{a|a \in T| f,(x a t)-f(x t) \mid<\epsilon$ for all $t \in T\}$. We prove

$$
A(\epsilon, f)=A^{-1}(\epsilon, f)
$$

and

$$
A^{2}(\epsilon, f) \subset A(2 \epsilon, f) \text {. }
$$

Let $a \in A(\epsilon, f)$, then $|f(x a t)-f(x t)|<\epsilon$ for all $t \in T$. Let $t^{\prime}=a t$ or $t=a^{-1} t^{\prime}$, then $\left|f\left(x a a^{-1} t^{\prime}\right)-f\left(x a^{-1} t^{\prime}\right)\right|<\epsilon$ or $\left|f\left(x t^{\prime}\right)-f\left(x a^{-1} t^{\prime}\right)\right|<\epsilon$ for all $t^{\prime} \in T$, whence $a^{-1} \in A(\epsilon, f)$. This completes the proof of (1).

Let $a, a^{\prime} \in A(\epsilon, f)$; then $\left|f\left(x a a^{\prime} t\right)-f(x t)\right| \leqq\left|f\left(x a a^{\prime} t\right)-f\left(x a^{\prime} t\right)\right|$ $+\left|f\left(x a^{\prime} t\right)-f(x t)\right|<\epsilon+\epsilon=2 \epsilon$. Thus $a a^{\prime} \in A(2 \epsilon, f)$. This completes the proof of (2).

Let $\epsilon>0$, then since $f_{x}$ and $g_{x}$ are discretely almost periodic, there exist $F=\left\{t_{i}\right\}_{i=1}^{n}$ and $G=\left\{s_{j}\right\}_{j=1}^{m}$, such that $A(\epsilon / 2, f) F=T$ and $A(\epsilon / 2, g) G=T$. Let $E_{i j}=\left[A(\epsilon / 2, f) t_{i}\right] \cap\left[A(\epsilon / 2, g) s_{j}\right]$, then $T$ $=\bigcup_{i=1}^{n} \bigcup_{j=1}^{m} E_{i j}$. Let $E=A(\epsilon, f) \cap A(\epsilon, g)$. Now for some $i$ and $j, E_{i j} \neq \varnothing$; thus let $u \in E_{i j}$. We prove $E u \supset E_{i j}$. Let $v \in E_{i j}$, then $v=a t_{i}$, since $E_{i j} \subset A(\epsilon / 2, f) t_{i}$. Now $u \in E_{i j}$, whence $u=a^{\prime} t_{i}$, where $a^{\prime} \in A(\epsilon / 2, f)$ or $t_{i}=a^{\prime-1} u$; therefore $v=a t_{i}=a a^{\prime-1} u$ or $v u^{-1}=a a^{\prime-1}$. But by (1) and (2), $[A(\epsilon / 2, f)]\left[A^{-1}(\epsilon / 2, f)\right] \subset A(\epsilon, f)$; therefore $v u^{-1} \in A(\epsilon, f)$. Similarly $v u^{-1} \in A(\epsilon, g)$, whence $v u^{-1} \in E$ or $v \in E u$. This completes the proof that $E u \supset E_{i j}$. Now for each $E_{i j} \neq \varnothing$ select $r_{k} \in E_{i j}$ and suppose there are $N$ such $r_{k}$. Then $U_{k=1}^{N} E r_{k}=\bigcup_{i=1}^{n} \bigcup_{j=1}^{m} E_{i j}=T$, and $E$ is discretely syndetic. Furthermore, by definition $E$ has the property that $b \in E$ implies $|f(x b t)-f(x t)|<\epsilon$ and $|g(x b t)-g(x t)|<\epsilon$ for all $t \in T$. This completes the proof of the lemma.

An application of Urysohn's lemma enables us to prove our last lemma.

3.7 Lemma. Let $X$ be a compact $T_{2}$-space; then for each index $\alpha$ of $X$ there exists a finite class of functions $\left\{f_{i} \mid i=1,2, \cdots, N\right\}$ on $X$ to the real numbers such that $\left|f_{i}(x)-f_{i}(y)\right|<1 / 2$ for $i=1,2, \cdots, N$ implies $x \in y \alpha$.

We are now in a position to prove the second half (sufficiency) of the principal theorem, 3.1.

Proof (sufficiency). We show $T$ is almost periodic. Let $\gamma$ be an index of $X$. By 3.7 there exists a finite class of functions $\left\{f_{i} \mid i=1,2, \cdots, n\right\}$ such that $\left|f_{i}(x)-f_{i}(y)\right|<1 / 2$ for $i=1,2, \cdots, n$ 
implies $x \in y \gamma$. By 3.5 there exist $A_{i}$ for each $i, 1 \leqq i \leqq n$, each syndetic in $T$, such that $\left|f_{i}\left(x A_{i} t\right)-f_{i}(x t)\right|<1 / 2$ for all $x \in X$ and for all $t \in T$. By 3.6 there exists a single $A$ such that $\left|f_{i}(x A t)-f_{i}(x t)\right|<1 / 2$ for all $i, 1 \leqq i \leqq n$, and for all $x \in X$ and all $t \in T$. Thus $x A t \subset x t \gamma$ for all $x \in X$ and all $t \in T$, whence $T$ is almost periodic. Finally by [2, Theorem 2], $T$ is equicontinuous. This complete the proof.

\section{REFERENCES}

1. H. Bohr, Almost periodic functions, New York, 1947.

2. W. H. Gottschalk, Almost periodicity, equi-continuity and total boundedness, Bull. Amer. Math. Soc. vol. 52 (1946) pp. 633-636.

3. W. H. Gottschalk and G. A. Hedlund, Topological dynamics (forthcoming).

4. A. Weil, Sur les espaces d structure uniforme et sur la topologie generale, Actualites Scientifiques et Industrielles, Paris, 1938.

YALE UNIVERSITY 